\title{
Laryngopharyngeal Reflux Alters Macrophage Polarization in Human Papilloma Virus-Negative Squamous Cell Carcinoma of the Larynx in Males
}

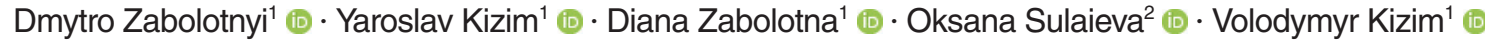 \\ ${ }^{1}$ Kolomiychenko Institute of Otolaryngology of National Academy of Medical Sciences of Ukraine, Kyiv; \\ ${ }^{2}$ Department of Histopathology, Laboratory of Pathology CSD, Kyiv, Ukraine
}

Laryngeal squamous cell carcinoma (LSCC) constitutes about $30 \%$ of all head and neck malignancies, and its incidence is increasing worldwide [1]. Although some risk factors, including smoking, alcohol consumption, human papilloma virus (HPV) infection, and radiation, are well established, the role of other potential risk factors remains controversial. The impact of laryngopharyngeal reflux (LPR) has attracted attention as a possible etiological factor of LSCC [2]. Long-term exposure of the laryngeal mucosa to gastric acid and enzymes due to LPR causes chronic inflammation, which is a defining feature of LPR-associated symptoms and can lead to precancerous transformation [3]. Other inflammatory conditions in different locations have been linked to the development of various malignancies and have been shown to affect the course and outcomes of cancer [4]. In this context, LPR can alter the tumor immune microenvironment (TIME), affecting the biological behavior and progression of LSCC [5]. Among the various cells involved in the TIME, macrophages have been shown to play a pivotal role in the prognosis of many malignancies, including LSCC.Although the link between LPR and LSCC is beyond doubt, it is still unknown whether and how LPR affects the TIME and macrophage polarization. In this study, we compared macrophage polarization in LSCC patients with and without coexisting LPR.

This pilot study was conducted at a single tertiary care center (the Kolomiychenko Institute of Otolaryngology of National Academy of Medical Sciences of Ukraine) in compliance with widely accepted guidelines for laryngeal cancer and LPR man-

\footnotetext{
- Received May 14, 2020

Revised July 15, 2020

Accepted July 17, 2020

- Corresponding author: Oksana Sulaieva

Department of Histopathology, Laboratory of Pathology CSD, Vasylkivska

st, 45, Kyiv 03022, Ukraine

Tel: +38-099-380-9417

E-mail: o.sulaieva@csd.com.ua
}

agement $[6,7]$. The study protocol was approved by the Institutional Ethical Committee (No. 11/17, 11.12.2017). All patients provided informed consent. The following inclusion criteria were used in the study: (1) histologically confirmed LSCC; (2) pT1 and pT2 tumors with no restrictions in terms of the tumor grade; (3) no adjuvant preoperative radiotherapy or chemotherapy at the time of diagnosis; and (4) negative results of HPV testing by polymerase chain reaction. Patients with recurrent cancer and those who used acid-suppressive medications were excluded from the study. A total of 63 patients with LSCC without (group 1, 30 patients; $63.1 \pm 1.42$ years old) and with coexisting LPR (group 2, 33 patients; $62.2 \pm 1.79$ years old) were enrolled in the study. The patients in groups 1 and 2 were matched in terms of age and tobacco smoking. LPR was diagnosed using the Koufman Reflux Symptom Index and the Reflux Finding Score based on findings during fiberoptic nasopharyngolaryngoscopy (Stryker90 endoscope with a Stryker SDC ULTRA HD informational manager; Stryker, San Jose, CA, USA), and proximal acid exposure percentage time by dual-probe $\mathrm{pH}$ monitoring (Acidogastrograph AG-1pH-M; Start LLC, Vinnytsia, Ukraine) [6]. The histological assessment included an investigation of tumor nests, as well as deep and peripheral margins. Immunohistochemistry was performed according to standard protocols using antibodies against CD68 (clone KP1; Dako, Santa Clara, CA, USA) and CD163 (clone MRQ-26; Cell Marque, Rocklin, CA, USA) to visualize M1 and M2 macrophages. The number of macrophages was counted in tumor clusters (TCs), tumor stroma (TS), and the intact laryngeal mucosa (IM) taken from the tumor-negative margins. The number of immunopositive cells was assessed per $1 \mathrm{~mm}^{2}$. In addition, the CD163/CD68 ratio, reflecting macrophage polarization, was evaluated. The blinded histological examination of the slides was performed by two independent observers (OS and $\mathrm{YK}$ ). The histopathological assessment was performed using Nikon Eclipse E200 microscopes (Nikon, Tokyo, Japan). Statisti-

Copyright @ 2021 by Korean Society of Otorhinolaryngology-Head and Neck Surgery.

This is an open-access article distributed under the terms of the Creative Commons Attribution Non-Commercial License (https://creativecommons.org/licenses/by-nc/4.0) which permits unrestricted non-commercial use, distribution, and reproduction in any medium, provided the original work is properly cited. 
cal analysis of the data was carried out with the MedCalc software (MedCalc Software, Ostend, Belgium). The descriptive statistics included the mean and standard error of the mean with $95 \%$ confidence intervals for continuous variables. The Student $t$-test was used to compare variables. Statistical significance was accepted for $P$-values $<0.05$.
No relationship was found between LPR and clinicopathological characteristics, including patients' age, tumor grade, or rate of keratinized and non-keratinized LSCC. The histological assessment revealed high-intensity inflammatory infiltration of both the tumor and unaffected mucosa at the peripheral margin of the resection in patients in group 2. Immune cells were rich in

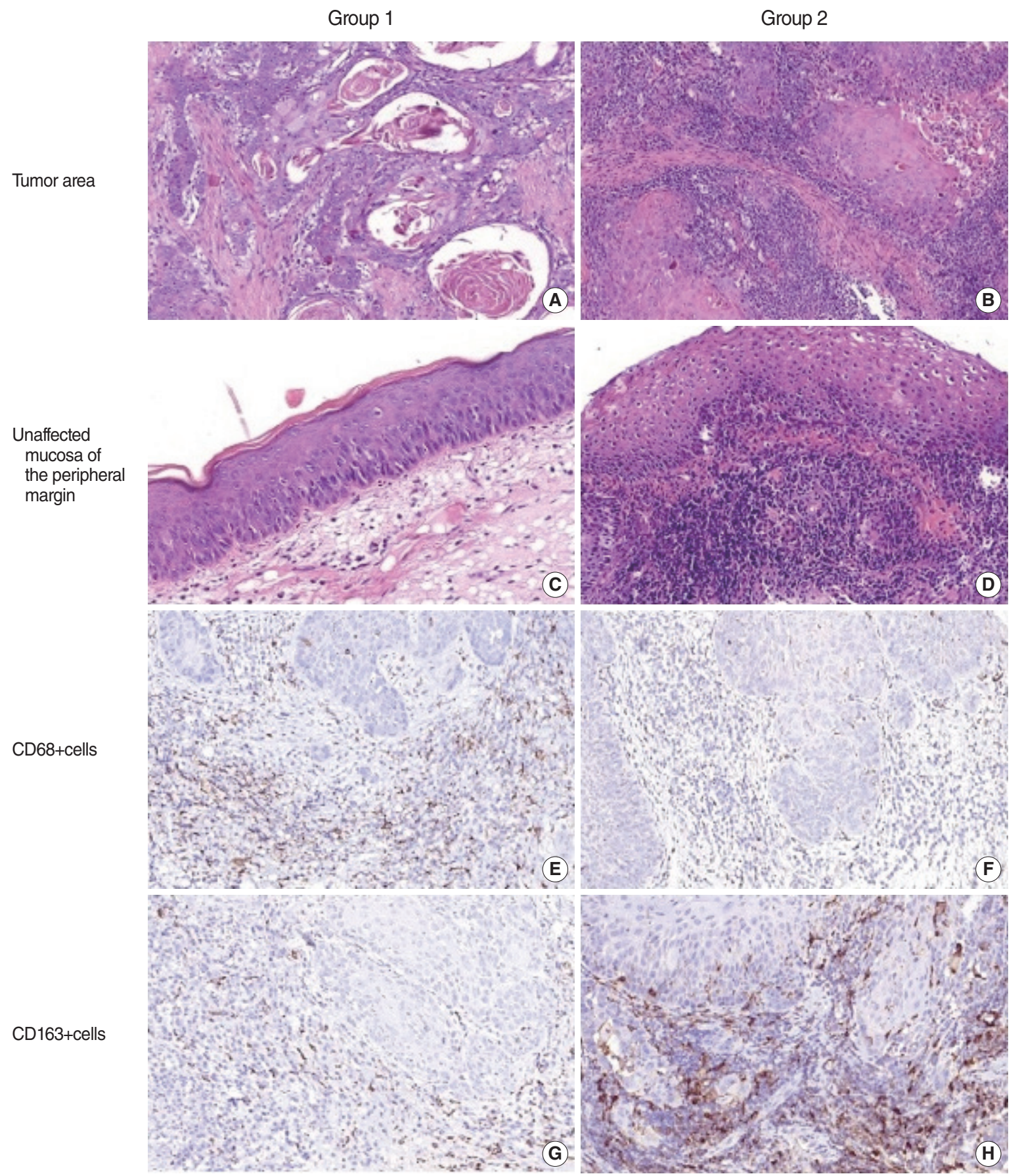

Fig. 1. Differences in inflammatory infiltration in patients with laryngeal squamous cell carcinoma (LSCC) with regard to laryngopharyngeal reflux (LPR). Group 1 included patients with LSCC without LPR, and group 2 comprised patients with LSCC+LPR. (A-D) Staining with H\&E. (E-H) Immunohistochemistry using antibodies against CD68 (E, F) and CD163 (G, H). Magnification, ×200. 
the TS and infiltrated TCs (Fig. 1). Macrophages of both phenotypes were more numerous in the invasive tumor margin than in the central tumor area in both groups. The number of M1macrophages was comparable in the TC of LSCC regardless of LPR. However, in group 1, CD68+ cells were much more numerous in the TS of LSCC. In contrast, there was a significant shift toward the M2-subtype of macrophages in the group 2 samples compared to group $1(P<0.001)$. The LPR-associated tumors demonstrated numerous CD163+ cells in both the epithelial fraction of LSCC and the TS. In addition, a significantly higher number of CD163+ cells was found in the IM of the larynx in group 2 than in group $1(P<0.001)$. Interestingly, $\mathrm{pH}$ values were negatively correlated with the amount of CD163 cells in group 2 , in both the TC $(r=0.71, P<0.001)$ and TS $(r=0.803$, $P<0.001)$. In addition, the CD163/CD68 ratio, an indicator of M2 polarization, was significantly higher in LSCC with coexisting LPR than in group $1(P<0.001)$ (Fig. 2$)$ Thus, LSCC with coexisting LPR showed a significant increase in M2 polarization.

The role of tumor-associated macrophages has been proven in many different malignancies. Macrophages can promote cancer progression and poor outcomes when alternative activation occurs, with polarization towards the M2 phenotype [8]. The protumor phenotype of macrophages can be affected by several aspects of the TIME, including cytokines, antibodies, lymphocytes, and tumor-derived chemokines [4,9]. In addition, changes in $\mathrm{pH}$ can impact tumor cells' proliferation and invasion ability, as well as the activities of immune cells [10]. Previous studies have demonstrated that acidosis alters macrophage phenotype characteristics, aggravating tumor progression [5,9]. However, no data have previously been reported on the impact of LPR on macrophage phenotypes in the larynx. In this study, we demonstrated that a decrease in laryngeal $\mathrm{pH}$ in patients with LPR was correlated with altered macrophage polarization. Indeed, LPRassociated LSCC environments were rich in M2 macrophages. Enhancement of the density of M2 macrophages is associated with an increase in the release of inflammatory and angiogenic cytokines/chemokines (e.g., vascular endothelial growth factor, CD14, and macrophage colony-stimulating factor) known to be
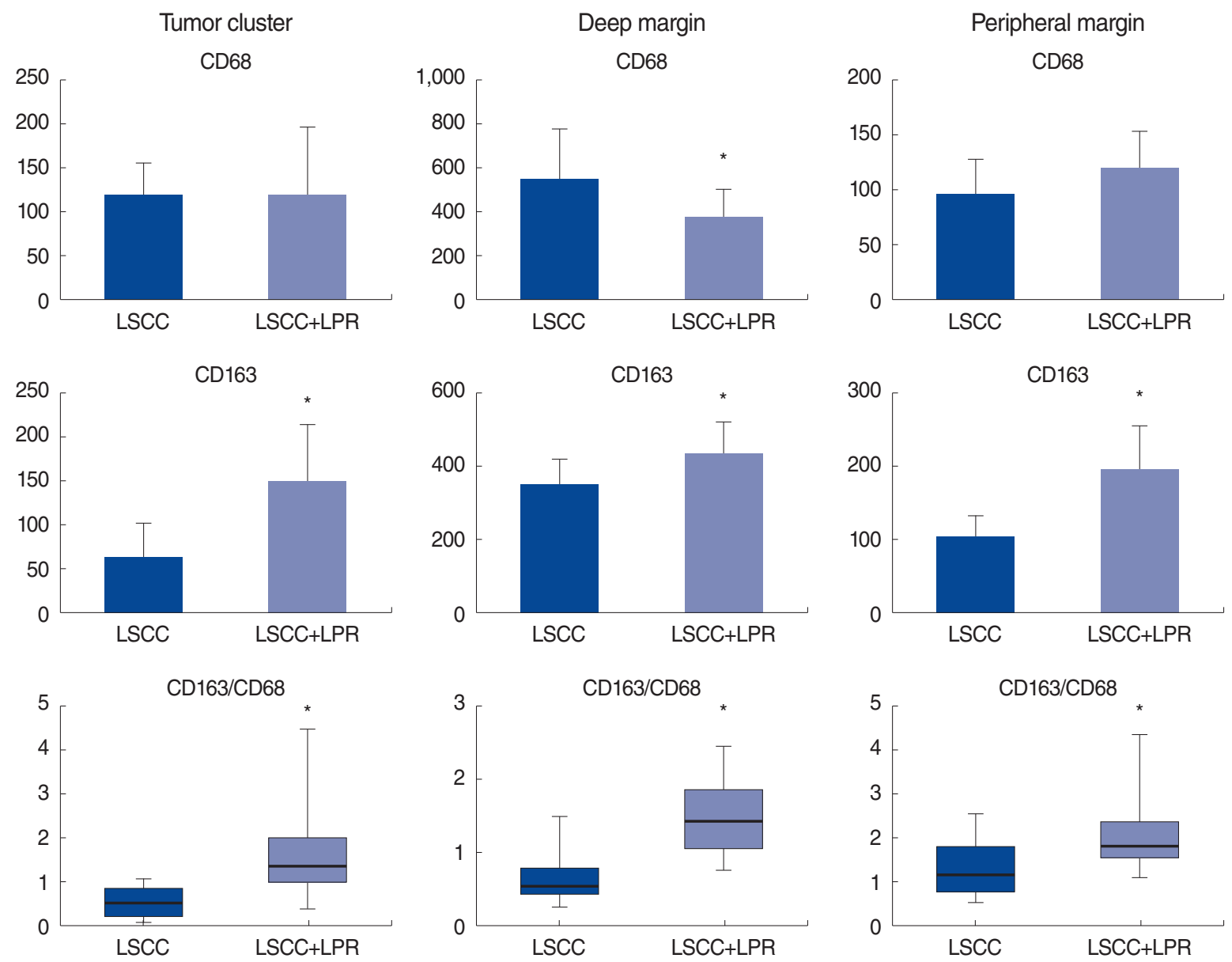

Fig. 2. Number of macrophage subtypes in different compartments of laryngeal squamous cell carcinoma (LSCC) according to the coexistence of laryngopharyngeal reflux (LPR). LSCC with coexisting LPR demonstrated a higher number of M2-type macrophages and a higher CD163/CD68 ratio in all compartments of the tumor. ${ }^{*} P<0.05$. 
involved in tumor progression [10]. Thus, LPR might initiate a scenario in which the acids generated by aspiration of gastric juice alter the phenotype of tumor-associated macrophages, creating a permissive niche for LSCC initiation and progression.

The evidence level of the results of this study is limited by the low number of patients and the lack of follow-up data. Nevertheless, the results of this study showed that LPR was associated with increased M2 polarization of macrophages, which may be a predisposing factor for LSCC development and progression.

\section{CONFLICT OF INTEREST}

No potential conflict of interest relevant to this article was reported.

\section{ORCID}

Dmytro Zabolotnyi

Yaroslav Kizim

https://orcid.org/0000-0001-9429-4414

Diana Zabolotna

https://orcid.org/0000-0001-5062-7707

Oksana Sulaieva

https://orcid.org/0000-0001-7807-8148

Volodymyr Kizim

https://orcid.org/0000-0002-9614-4652

https://orcid.org/0000-0002-9611-4998

\section{AUTHOR CONTRIBUTIONS}

Conceptualization: VK, OS, DZ (Dmytro Zabolotnyi). Data curation: DZ (Diana Zabolotna), YK. Formal analysis: DZ (Diana Zabolotna), YK. Methodology: DZ (Dmytro Zabolotnyi), VK. Project administration: VK, OS. Visualization: OS. Writing-origi- nal draft: OS, YK, DZ (Diana Zabolotna). Writing-review \& editing: DZ (Dmytro Zabolotnyi), VK.

\section{REFERENCES}

1. Sandulache VC, Wilde DC, Sturgis EM, Chiao EY, Sikora AG. A Hidden epidemic of "intermediate risk" oropharynx cancer. Laryngoscope Investig Otolaryngol. 2019 Oct;4(6):617-23.

2. Tae K, Jin BJ, Ji YB, Jeong JH, Cho SH, Lee SH.The role of laryngopharyngeal reflux as a risk factor in laryngeal cancer: a preliminary report. Clin Exp Otorhinolaryngol. 2011 Jun;4(2):101-4.

3. Greten FR, Grivennikov SI. Inflammation and cancer: triggers, mechanisms, and consequences. Immunity. 2019 Jul;51(1):27-41.

4. Binnewies M, Roberts EW, Kersten K, Chan V, Fearon DF, Merad M, et al. Understanding the tumor immune microenvironment (TIME) for effective therapy. Nat Med. 2018 May;24(5):541-50.

5. Boedtkjer E, Pedersen SF. The acidic tumor microenvironment as a driver of cancer. Annu Rev Physiol. 2020 Feb;82:103-26.

6. Koufman JA, Aviv JE, Casiano RR, Shaw GY. Laryngopharyngeal reflux: position statement of the committee on speech, voice, and swallowing disorders of the American Academy of OtolaryngologyHead and Neck Surgery. Otolaryngol Head Neck Surg. 2002 Jul; 127(1):32-5.

7. Korean Society of Thyroid-Head and Neck Surgery Guideline Task Force, Ahn SH, Hong HJ, Kwon SY, Kwon KH, Roh JL, et al. Guidelines for the Surgical Management of Laryngeal Cancer: Korean Society of Thyroid-Head and Neck Surgery. Clin Exp Otorhinolaryngol. 2017 Mar;10(1):1-43.

8. Qian BZ, Pollard JW. Macrophage diversity enhances tumor progression and metastasis. Cell. 2010 Apr;141(1):39-51.

9. Corbet C, Feron O.Tumour acidosis: from the passenger to the driver's seat. Nat Rev Cancer. 2017 Oct;17(10):577-93.

10. El-Kenawi A, Gatenbee C, Robertson-Tessi M, Bravo R, Dhillon J, Balagurunathan $Y$, et al. Acidity promotes tumour progression by altering macrophage phenotype in prostate cancer. Br J Cancer. 2019 Oct;121(7):556-66. 